\title{
Subject Age in P300 BCI
}

\author{
N. S. Dias, P. M. Mendes, J. H. Correia \\ Dept. Industrial Electronics, University of Minho \\ Campus Azurem, 4800-058 Guimaraes, Portugal \\ ndias@dei.uminho.pt
}

\begin{abstract}
This paper presents a study about the amplitude, latency and distribution of $\mathrm{P300}$ in twelve healthy volunteers (5 women and 7 men, aged between 19-70, with a mean of 35.5 years) that were submitted to two Novelty Oddball Paradigms. The difference between them is on stimulus modalities, which are visual and auditory. Usually, a P300-based brain computer interface has visual stimuli but we are also interested in making analogies between the two modalities. In both test modalities were presented 3 types of stimuli: a 'standard' stimulus, a 'target' stimulus, to which the subject must respond, and a 'novel' stimulus. Most of the subjects were students and are naive to the purposes of the experiment. The subject is not warned about the 'novel'. We recorded scalp potentials from 18 channels distributed in a standard configuration 10-20 system. Target P300 had more relevance than novel P300 for a BCI application. Results show relevant differences in amplitude and latency in P300 signal for different ages. The Older subjects ( $>51$ years) show smaller P300 amplitudes than younger. Therefore, the subject age must be taken into account when a P300 BCI application is implemented.
\end{abstract}

\section{INTRODUCTION}

A brain computer interface $(\mathrm{BCI})$ is a real-time communication system designed to allow users to voluntarily send messages or commands without sending them through the brain's normal output pathways [1]. A BCI device allows people to communicate without movement. People can send information simply by thinking. Everybody can imagine how useful would be a system that could know accurately what we desire to do just by reading our scalp potentials. BCI users must produce distinct EEG signatures when engaged in discrete mental tasks originated by visual or auditory stimuli. P300 test could be the trigger of these tasks. In a P300 test we don't analyze the free running electroencephalogram (recorded EEG) but the event related potential (ERP). ERPs are voltage fluctuations in the EEG induced within the brain that are time locked to sensory, motor, or cognitive events [2]. The ERPs consist of a sequence of positive and negative voltage fluctuations. These components reflect various sensory, cognitive (e.g., stimulus evaluation) and motor processes that are classified on the basis of their scalp distribution and response to experimental variables [2]. The $\mathrm{P} 300$ component is a positive bump in the ERP that typically starts around $300 \mathrm{~ms}$ after an event and reflects a cognitive process about the deviant event (an event highlighted from the background events). Mismatch Negativity (MMN) component is a negative bump in the ERP that starts before $250 \mathrm{~ms}$ after the event presentation and reflects the response to the difference between successive stimuli [3]. The deviant event is highlighted by its physical differences (e.g. frequency tone in auditory modality) and occurrence probability difference related to the background event (standard stimulus). Therefore, it can be an expected event (response-requiring or target stimulus) or a non-expected event (novel event). Novel events response depends on familiarity, habituation and semantic aspects related to the stimuli [2]. Subject's nature and stimulus' parameters differences can produce a large variety of P300 type waves that represent different neural processes. On one hand, we can manipulate stimulus parameters (e.g. figures' contrast in visual modality) in order to get P300 components easily recognizable by the BCI system. On the other hand, we can try to attenuate subject's nature effects on P300. Subject's nature depends on age. Therefore, it's very important to know what are the real effects of age on target P300 as an ERP and on subject's behaviour (performance in response to target) during these tasks. There are few factors that lead few BCI users to better performing stimuli detection than others.

The factor under work is the age and it's thought to increase P300 latency and to reduce P300 amplitude [4] as well as behavioural response impairment. In literature, we found many studies about P300 age effect, but almost all of them gave more importance to P300 elicited by novel events. We pretend in this study to acknowledge these hypotheses to target P300 in both visual and auditory modalities and with some variability in stimuli-type.

\section{TESTS DESCRIPTION}

Usually the P300 is elicited during an Oddball Task. This component can be seen after the occurrence of any stimulus but it's highlighted in the presence of a low frequent stimulus (target) that demands subject attention.

In this study were used 2 test modalities. A Novelty Oddball Paradigm with auditory stimuli, similar to the one used in [5] and a Novelty Oddball Paradigm with visual stimuli. The Novelty Oddball Paradigm is a kind of paradigm in which 3 classes of stimuli were delivered to the subject. In the auditory modality, a 'standard' high-probable $(\mathrm{P}=0.80)$ stimulus, a 'target' low-probable $(\mathrm{P}=0.10)$ stimulus and a 'novel' lowprobable $(\mathrm{P}=0.10)$ stimulus were presented. Each subject was instructed about 'standard' and 'target' stimuli (response by clicking on the left mouse button) but wasn't about 'novel'. When the subject asked about the 'novel', he/she was just instructed to continue the test. The standard stimulus is a pure tone of $500 \mathrm{~Hz}$ with duration of $336 \mathrm{~ms}$, the target stimulus is a pure tone of $350 \mathrm{~Hz}$ with duration of $336 \mathrm{~ms}$ and the novel 
stimuli are 30 unclassified sounds. Each trial lasts $1000 \mathrm{~ms}$ and starts with the stimulus. In theory, we have 3 kinds of trials, one for each stimulus type. The trials are presented in blocks and each one comprises 150 trials in which the subject should respond to 'target' stimuli as fast as he/she can but also in an accurate way.

EEG data are recorded during 6 blocks of trials in order to get an ERP optimum average (about 80 samples removing artifacts) because we just have 15 samples of target P300 per block. The stimuli delivered as novels are unique in each block of trials and none of them will be repeated within a block. The EEG data will be recorded on the following electrode sites according the 10-20 system: VEOG (Vertical EOG), HEOG (Horizontal EOG), F7, F3, Fz, F4, F8, FC1, FC2, T7, Cz, T8, Tp9, Tp10, P7, Pz and P8 referenced to A (linked earlobes). VEOG is a bipolar channel placed on the left eye to record vertical electrooculogram data and HEOG is also a bipolar channel placed on both eyes outer canthi. These 2 channels are used for ocular artifact removal. All the others are single channels and some of them are used just for permitting better topographical maps.

The visual modality test is equal to the auditory one unlike some aspects that will be enumerated. The standard has an occurrence probability of 0.76 , the target and novel stimuli have a probability of 0.12 . This test has 2 branches in respect to the stimuli: human faces and geometrical shapes. In the first, the standard stimulus is a man face, the target is a woman face and the novel is a face among 30 faces of men or women in different mood. In the second, the standard is a simpler geometrical shape, the target is more complex than standard and novel is a shape among 30 very complex geometrical shapes. Each stimulus lasts $200 \mathrm{~ms}$. Each block comprises 250 trials and EEG data are recorded during 3 blocks. The recording sites are: VEOG, HEOG, F7, F3, Fz, F4, F8, FC1, FC2, T7, Cz, T8, Tp9, Tp10, P7, Pz, P8, O1, Oz and O2 referenced to A (linked earlobes). The subjects stayed $1 \mathrm{~m}$ far away from the stimuli presentation device.

We used faces and shapes as stimuli in the visual test in order to find possible dependencies between stimuli nature and P300 variability.

\section{HARDWARE SYSTEM}

The hardware system is composed by an amplifier with 40 channels and A-to-D conversion of 22-bits (sampling frequency at $2000 \mathrm{~Hz}$ ), sintered $\mathrm{Ag} / \mathrm{AgCl}$ ring electrodes for guaranteeing low constant transition resistance and a braincap with large filling holes and flat clip on adapters making skin preparation and gel application simpler and improving preparation time. Meanwhile, we are developing a wireless braincap with integrated antenna [6].

The amplifier is connected to a PC (via USB) that runs a recording software. A computer 15"display was used, $1 \mathrm{~m}$ far away from the subject, connected to amplifier via parallel port to make stimuli/data synchronization. The auditory stimuli were presented to subject with headphones. The entire system is used like in Fig. 1.

\section{EXPERIMENTAL RESULTS}

P300, as an ERP waveform, can be characterized quantitatively in order to peak amplitude, peak latency and scalp distribution.

As all the twelve subjects are between 19-70 and we pretend to evaluate age effects, they were equally distributed in three age classes (4 subjects per class). The first one is ranging between 19-30, the second one between 31-50 and the third one between 51-70. The younger ones will be compared just with the older ones for all presented results and it's assumed that the middle-age class results are between them.

The recorded data are filtered with Butterworth zero phase filters: a low cut-off of $0.5 \mathrm{~Hz}, 48 \mathrm{~dB}$ /oct and a high cut-off of $35 \mathrm{~Hz}, 48 \mathrm{~dB} /$ oct. A Notch filter of $50 \mathrm{~Hz}$ was used to reduce the electrical noise. After filtering, ocular correction was done off-line with the algorithm Gratton and Coles [7]. The channels used for ocular correction were VEOG to identify vertical artefacts and HEOG to identify horizontal artefacts. Then, data were segmented according stimuli-type. Each epoch starts $100 \mathrm{~ms}$ before the stimulus presentation and ends $800 \mathrm{~ms}$ after stimulus. Each stimulus-type segment was inspected for other type of artefacts. An average of all segments (within a block) of a particular stimulus-kind was done. For example, a target average results from the average of all target stimulus segments within a block. The ERP, also called Grand Average ERP, is the average of all blocks averages for a particular stimulus-kind.

The analysed ERPs are always the difference between the Grand Average ERP we pretend to survey (target ERP or novel ERP) and the Grand Average ERP of the standard stimulus. The P300 component was seek in the ERP as the most positive point between 250 and $600 \mathrm{~ms}$ and the MMN component as the most negative point between 0 and $250 \mathrm{~ms}$ after stimulus presentation.

There are some evident results within an age class. The MMN component always shows smaller amplitudes in target ERP than in novel ERP for every age class and every test. MMN has a distribution central-oriented in auditory tests and parietal-oriented in visual tests for every age class.

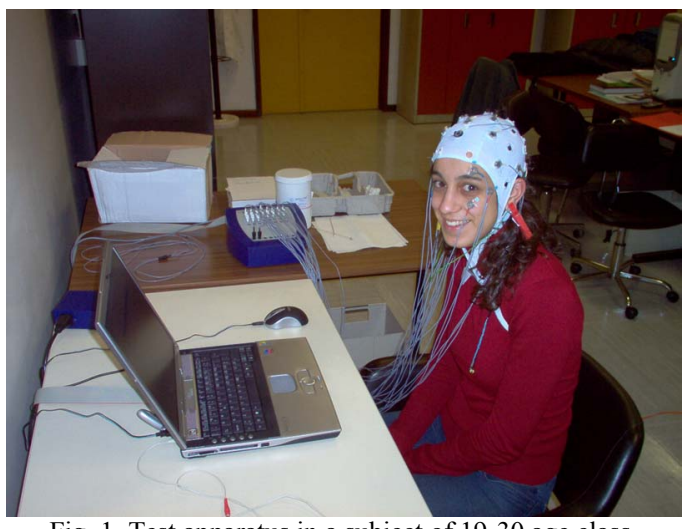

Fig. 1. Test apparatus in a subject of 19-30 age class. 
In the 19-30 age class, the target P300 component shows a parietal distribution in the auditory tests and a distribution parietal-oriented in visual tests. In the oldest class, the target P300 component has a parietal distribution, with large components at frontal sites, for the auditory tests and a distribution parietal-oriented, with large components at central sites, for the visual tests (see Fig. 2). The target P300 amplitude is typically largest over the Pz site.

About auditory tests, 19-30 age class show MMN components of smaller amplitudes and P300 components of bigger amplitudes than the oldest age class (see Fig. 2). Although of almost no evidence, the P300 components of the youngest seem to have an upper latency than the oldest ones. MMN components latencies of both age classes look very similar.

About visual tests, 19-30 age class target P300 components have bigger amplitudes and smaller latencies (for faces stimuli) or bigger latencies (for shapes stimuli) than the oldest one. The youngest have MMN components with less amplitude and latency than the oldest ones (see Fig. 2).

From literature, the novel $\mathrm{P} 300$ has a distribution, over the entire scalp, with frontally prominence and the target P300 has a posterior distribution as confirmed by these results [5]. Oldest subjects ( $>51$ years) show smaller P300 amplitudes, bigger P300 latencies than younger in visual faces stimuli, smaller in visual shapes stimuli and almost equal in auditory stimuli.

\section{DISCUSSION AND CONCLUSIONS}

About the results within an age class, the expected lower MMN amplitudes in target ERP than in novel ERP are due to the fact that MMN is the response to the difference between successive stimuli. Therefore, the target stimulus is less different from standard than the novel. The MMN is thought to be a component frontally oriented in general but it's also usually associated to a sensory-specific generator. Then, it's normal that in the auditory test, the MMN is large on $\mathrm{Cz}$ (the site nearest to the primary auditory cortex). Also, in the visual tests, the MMN is larger on $\mathrm{Pz}$ (the site nearest to the primary visual cortex on the occipital).

The 19-30 age class shows a target P300 component larger over parietal sites, for auditory and visual tests, as well as the oldest class, but the last one shows also large components over frontal and central sites. In literature, the frontal P300 components reflect a stimulus memory template creation/management [2]. The target stimulus is previously known by the subject, then it's not supposed to need a template creation/management but a constantly categorization. The posterior P300 components reflect stimulus categorization, as referred in literature [2]. Therefore, the target P300 is typically larger over parietal sites. The large frontal components of the oldest class may represent an additional need of stimuli memory template creation/management due to a lack of habituation. a) Auditory grand average

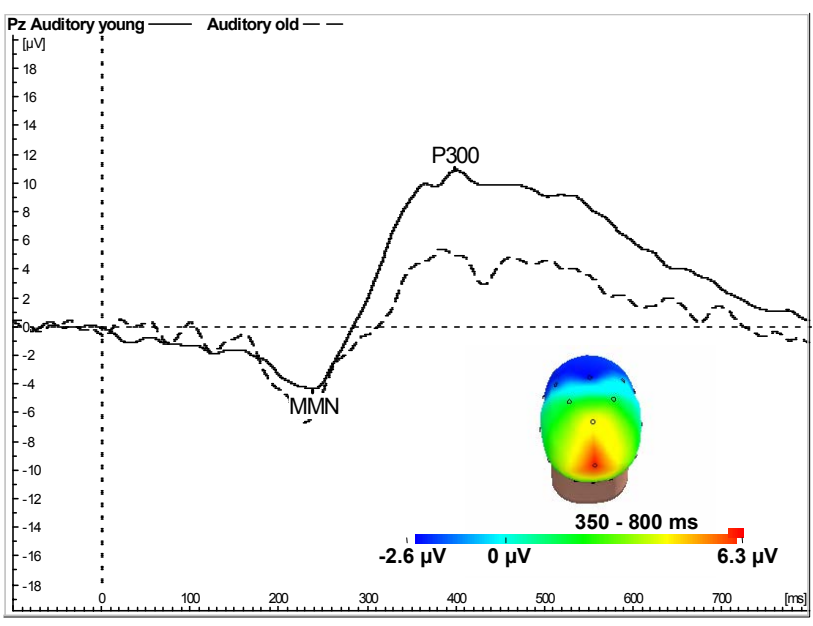

b) Faces stimuli visual grand average

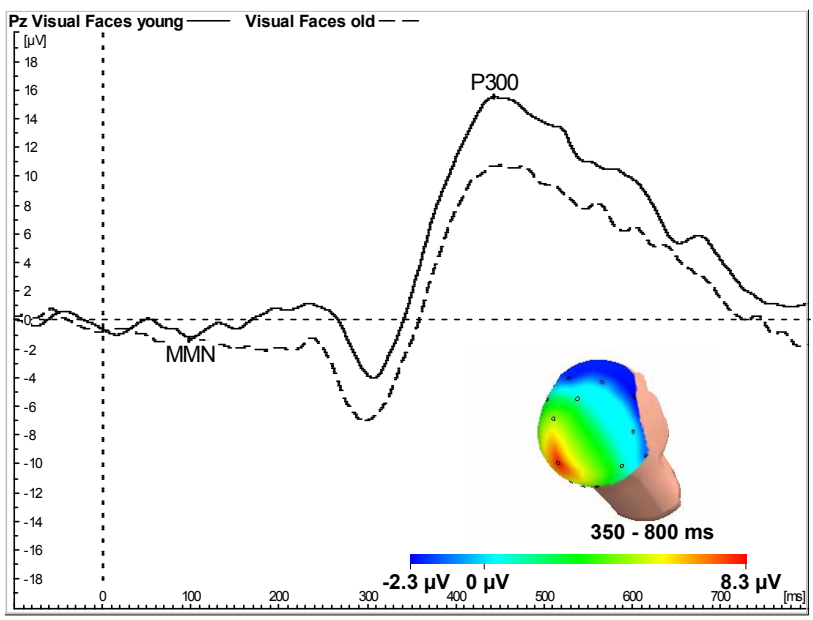

c) Shapes stimuli visual grand average

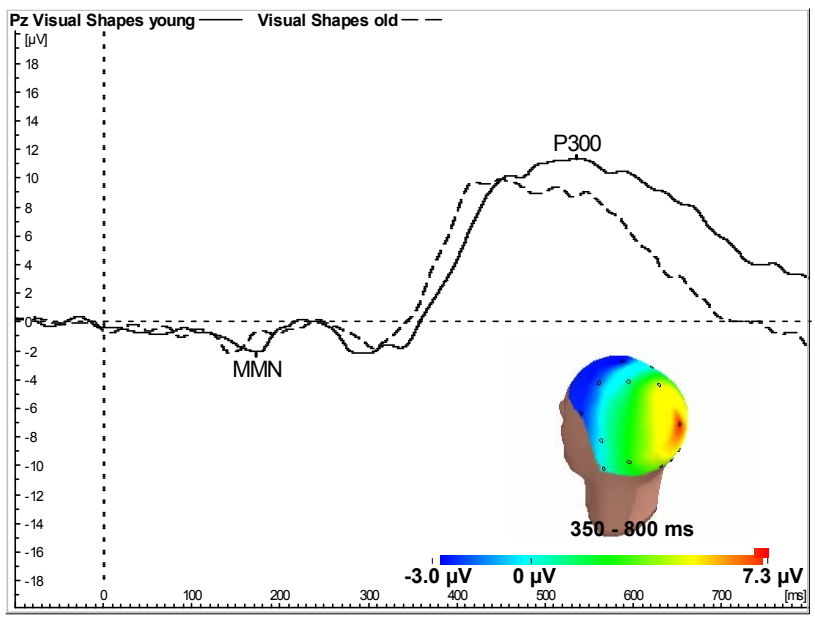

Fig. 2. Comparison of the 3 types tests grand averages for the youngest subjects (solid line) and the oldest subjects (dashed line) in waveform view over Pz site (Time on X-axis and voltage potential on Y-axis). Potentials scalp distribution 3D mapping view for youngest subjects. 
Habituation is well known as an amplitude decrease of controls (subjects with no impairments) to frontal P300 components with experience [8]. This extra need can suggest progressive frontal cognitive functions impairment with age and elderly lack of habituation.

The results don't support the P300 latency increase with age. Younger P300 components should have lower latencies than older ones. In auditory modality the younger P300 latency doesn't agree with this latency hypothesis. In visual modality the P300 latencies support the latency hypothesis for faces stimuli and does not for shapes stimuli. The latency reveals the timing of the larger neural activation [2]. These unexpected results can be corrected, as well as more subjects data will be added in future scheduled tests. Therefore, if the faces stimuli show expected results and shapes stimuli unexpected results, then stimuli-type variability may induce variability results.

The youngest class has smaller MMN amplitudes and bigger target P300 amplitudes than the oldest one for both test modalities. This fact supports the target P300 amplitude age effects hypothesis. Amplitude provides an index of the extent of neural activity [2]. The target P300 amplitude decrease for older can be related to neural activity reduction with age and MNN amplitude increase may be related to the successive stimuli differences sensitive increase, as Gaeta and Friedman concluded [9].

We have demonstrated that the subject age must be taken into account when a P300 BCI application is implemented. Results show relevant differences in amplitude P300 signal for different ages and latency variability for stimuli-type variability. Older subjects ( $>51$ years $)$ show smaller amplitudes than younger subjects on target P300 at Pz site. Therefore, age effects on P300 components can be compensated toward an age effects immune BCI. Preprocessing techniques like ICA [10] and other pattern recognition techniques can attenuate $\mathrm{P} 300$ age effects.

\section{REFERENCES}

[1] B. Allison, PhD Thesis, "P3 or not P3: Toward a Better P300 BCI," University of California, San Diego, 2003.

[2] D. Friedman, Y. M. Cycowicz and H. Gaeta "The novelty P3: an eventrelated brain potential (ERP) sign of the brain's evaluation of novelty," Neuroscience and Biobehavioral Reviews, vol. 25, 2001, pp. 355-373.

[3] A. Mecklinger, B. Opitz, A.D. Friederici, "Semantic aspects of novelty detection in humans," Neurosciences Letters, vol. 235, 1997, pp. 65-68.

[4] D.S. Goodin et al., "Auditory evoked-potentials in aging and dementia," Neurology, vol. 28, no. 4, 1978, pp. 385-385.

[5] M. Fabiani and D. Friedman, "Changes in brain activity patterns in aging: the novelty oddball," Psychophysiology, vol. 32, 1995, pp. 579-594.

[6] P.M. Mendes, A. Polyakov, M. Bartek, J.N. Burghartz, J.H. Correia, "An Integrated Folded-Patch Antenna for Wireless Microsystems," Proc. of IEEE Sensors 2004, 24-27 October 2004, Vienna, Austria.

[7] G. Gratton, M.G.H. Coles, and E. Donchin, "A new method for off-line removal of ocular artefact," Electroencephalography and Clinical Neurophysiology, vol. 55, 1983, pp. 468-484.

[8] D.Friedman et al., "Effects of aging on the novelty P3 during attend and ignore oddball tasks," Psychophysiology, vol. 35, 1998, pp. 508-520.

[9] H. Gaeta et al., "An Event-Related Potential Evaluation of Involuntary Attentional Shifts in Young and Older Adults," Psychology and Aging, vol. 16, no.1, 2001, pp. 55-68.

[10] N. Xu, X. Gao et.al., "BCI Competition 2003 - Data Set IIb: Enhancing P300 Wave Detection Using ICA-Based Subspace Projections for BCI Applications," IEEE Trans. Biomed. Eng., vol. 51, no. 6, June 2004, pp. 1067-1072. 